\title{
Mission Planning and System Design for HISA: A Hyperspectral Imaging Satellite for Australia
}

\author{
By Eugene KIM, ${ }^{1)}$ Xiaofeng WU, ${ }^{1)}$ and Warwick HOLMES ${ }^{1)}$ \\ ${ }^{1)}$ School of Aerospace, Mechanical and Mechatronics Engineering, The University of Sydney, Sydney, Australia
}

(Received June 23rd, 2017)

\begin{abstract}
The School of Aerospace, Mechanical and Mechatronics Engineering (AMME) at The University of Sydney is proposing the design of a commercially viable $100 \mathrm{~kg}$ class microsatellite, HISA - a Hyperspectral Imaging Satellite for Australia. The primary mission is centred around the application of hyperspectral imagery to address compelling agricultural and environmental problems specific to Australia at a low cost. This study presents the technical mission requirements for maximum utility and data throughput. It also presents a description of system design and the subsystems which have been carefully selected after discussions with Australian Government departments.
\end{abstract}

Key Words: Hyperspectral, Microsatellite, Remote Sensing, Systems Engineering, Earth Observation

\section{Introduction}

Hyperspectral imagery is able to provide the interaction of electromagnetic radiation with an object over hundreds of narrow continuous bands of the electromagnetic spectrum. Normal optical cameras provide information in the RGB spectrum and can distinguish between different objects such as rocks, trees, and water. However, hyperspectral imagery is able to provide the composition of objects and can discriminate between different types of rocks and trees by measuring the reflectance, emission and absorption of electromagnetic radiation in each pixel. Each image consists of two spatial dimensions and one spectral dimension, forming a hyperspectral 'datacube' as shown in Fig. 1. Thus it has become one of the most information rich sources of data which is growing in significance in many research areas. Hyperspectral imagery can be applied to areas such as precision agriculture, land cover identification and mineral mapping.

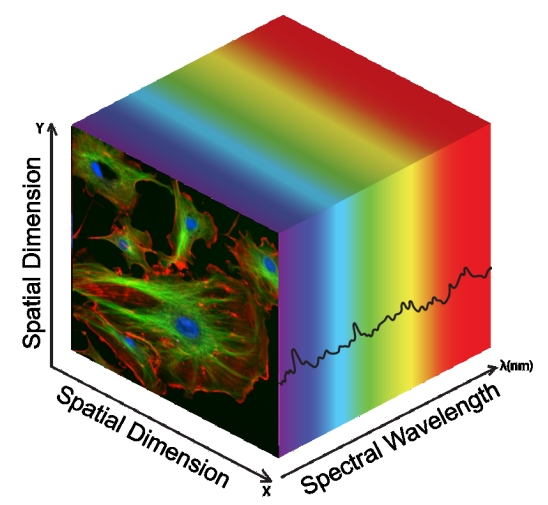

Fig. 1. A hyperspectral image datacube consists of two spatial dimensions and a spectral dimension comprised of many bands.

Before space-based hyperspectral imaging platforms, airborne sensors were used to acquire accurate images with high spatial and spectral density. The first airborne hyperspectral system to fly was the Airborne Imaging Spectrometer (AIS) by NASA in 1984. ${ }^{1)}$ Several other notable airborne hyperspectral systems include HyMap, CASI and AVIRIS. Although airborne platforms are able to target specific areas with greater accuracy and resolution than space-based platforms, it tends to have a lower swath width and thus is not viable in covering a wide area.

Hyperspectral imaging platforms mounted onboard satellites can cover a wide area in a very short period whilst maintaining a revisit time of a few days. It is also cheaper to operate than airborne systems (\$0.09 USD/ha compared to $\$ 1-2$ $\mathrm{USD} /$ ha for airborne imagery; data based on Hyperion operation $\operatorname{costs^{2})}$ ). Spaceborne hyperspectral imaging sensors and their respective spacecraft include CHRIS (PROBA-1, ESA), Hyperion (EO-1, NASA), and ARTEMIS (TacSat-3, AFRL). More recently, hyperspectral sensors which are currently under development include HISUI (ALOS-3, JAXA), PRISMA (Italian Space Agency ASI), HyspIRI (NASA) and EnMAP (DLR).

Current trends in space research and development has seen space agencies and organisations place greater emphasis on reducing the costs involved in the development and operation of satellites. Advances in technology has seen the increase in development of small satellites. Microsatellites are a class of small satellites with a mass ranging between 10-100 $\mathrm{kg}$ and are increasing in popularity due to the lower development costs, high reliability compared to larger satellite platforms whilst demonstrating the capability of performing complex operations. SpaceWorks has forecasted an estimated 182 nano/microsatellites to launch in 2017 , an $80 \%$ increase from 2016. ${ }^{3)}$

The School of Aerospace, Mechanical and Mechatronics Engineering (AMME) at The University of Sydney (USYD) is proposing the system design and mission planning for a $100 \mathrm{~kg}$ class microsatellite "HISA" - a Hyperspectral Imaging Satellite for Australia. It aims to provide dedicated coverage of Australian land and waters and provide both hyperspectral and high resolution images to government departments such as Commonwealth Scientific and Industrial Research Organisation (CSIRO) and to private organisations who may be able to benefit from access to readily available satellite imagery.

This study presents a preliminary system design of the first commercially viable microsatellite platform with hyperspec- 
tral and high resolution imaging capabilities dedicated to Australian land and agriculture. Although there have been previous and upcoming missions which utilise hyperspectral imagery for Earth observation (EO), HISA aims to demonstrate the novelty of specific system engineering optimisation in all subsystems to optimise temporal-spatial-spectral hyperspectral imagery. This includes:

- Optimisation of the hardware and software to fit in the volume and form factor of a $80 \times 80 \times 100 \mathrm{~cm}$ microsatellite platform.

- High throughput data downlink capacity using an X-band phased array antenna with high capacity QAM scheme.

- High attitude pointing accuracy with "nodding" to increase integration time with autonomous pointing profiles and target acquisition.

- Autonomous level-0 onboard image processing including cloud detection, bad-pixel map, image sensor calibration pre-processing to improve pipeline-image processing.

Integration of all the features mentioned above in the volume of a microsatellite constrains the technology and hardware that can be used for this specific hyperspectral application. The satellite falls between the conventional small sized $(100-500 \mathrm{~kg})$ remote sensing satellite and much smaller cubesats (1-10 kg). The design shall include specific optimisation in onboard autonomy in terms of satellite bus operation, payload image pre-processing, ground segment mission planning, and distribution and fusion of metadata providing autonomous distribution of image data.

This paper first introduces applications of hyperspectral imagery for Australia and presents a detailed description of the primary payload requirements which were identified and outlined by multiple potential customer bases in Australia. It is followed by the mission planning and details of the overall system design of the subsystems.

\section{Hyperspectral Imagery in Australia}

Currently, Australia is one of only two countries in the Organisation for Economic Cooperation and Development $(\mathrm{OECD})^{4)}$ to lack a dedicated space agency and resorts to outsourcing development of spacecraft and launch services to other nations at a high cost. Also, Australia does not currently own any satellites with EO or remote sensing capabilities and must rely on acquiring images from other nations; the estimated government expenditure on acquiring remote sensing data was approximately $\$ 100$ million per annum in 2012.5) EO data downloads (free and purchased) used for Australian research projects exceeded 100 TB in 2012 and is estimated to have exceeded 1 PB in 2016. ${ }^{6}$

It is evident that the use of hyperspectral data is becoming an integral part of Australian research. However, the application of hyperspectral imagery is very broad and thus, major Australian government departments (CSIRO, Geoscience Australia, Department of Agriculture and Water Resources, Australian Bureau of Agricultural and Resource Economics and Sciences) were contacted to identify which specific applications of hyperspectral imagery is of greatest importance to the Australian research community. From this information, technical specification requirements for the hyperspectral camera and spacecraft system are established. Some Australian applications include precision agriculture, mineral mapping, land cover identification and water quality monitoring. In particular, hyperspectral monitoring of water toxicity caused by harmful algal blooms in inland water bodies takes priority.

Blue-green algae is a type of bacteria called cyanobacteria which is capable of producing toxins, and affect water which is used for drinking, agriculture and recreation. It drastically reduces water quality and is fatal to fish, domestic animals, and is harmful to human health. It is caused by a range of conditions including: low water turbidity, warm water temperature and high concentrations of phosphorus and nitrogen. It is a growing threat to inland water bodies in Australia; the MurrayDarling Basin (MDB) is an area of particular concern regarding this issue. In 1991, over $1000 \mathrm{~km}$ of the Darling River was covered in blue-green algae and was the largest algal bloom ever recorded (Fig. 2). It had catastrophic impact to the environment, killing native fauna, livestock and vegetation. It was also affected agricultural production and was a big blow to the Australian economy as the MDB accounts for $39 \%$ of the total Australian income for agricultural production and produces over a third of Australia's food supply. ${ }^{8)}$ Other instances of algal blooms occurred in Murray River in 2007, 2009, 2010, and 2016.

The Australian Government estimates the cost of algal blooms to be between $\$ 180-240$ million AUD $^{8)}$ for anticipated costs in environmental protection, and management and extraction. Traditional methods of testing for algal blooms include travel to locations which are not easily accessible and involve costly and lengthy tests in a laboratory. Thus, is essential to have remote sensing capabilities to detect early stages of algal blooms to prevent the environmental, agricultural and socioeconomic impact on Australia.

Previous research in algal bloom detection has pointed towards the identification of chlorophyll-a and phycocyanin levels in water. Studies have shown that chlorophyll-a has a distinct absorption peak at $430 \mathrm{~nm}$ and $660 \mathrm{~nm}^{9)}$ while phycocyanin peaks in absorption at $620 \mathrm{~nm} .{ }^{10}$ ) Thus, the absorption and reflectance will be measured at but not limited to these wavelengths.

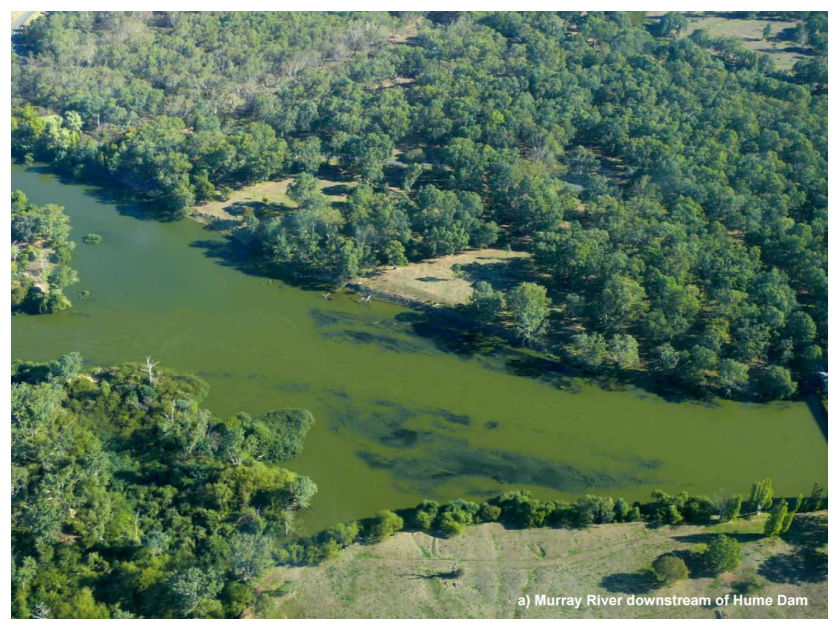

Fig. 2. A part of the Murray River affected by blue-green algae known as cyanobacteria. This affects water used for agriculture, drinking and recreation. $^{8)}$ 


\section{Mission Planning}

There are two scientific missions revolving around the primary and secondary payload. The primary mission aims to acquire hyperspectral imagery data of the Earth's surface - in particular the Visual and Near-InfraRed (VNIR) and Short-Wave InfraRed (SWIR) wavelengths. A secondary EO mission is also implemented to further realize the remote sensing capabilities of HISA. The mission planning also consists of the orbit analysis and launch opportunities.

\subsection{Primary mission}

The primary scientific mission revolves around the main payload - the hyperspectral camera and can be explicitly stated as:

"The acquisition of hyperspectral imagery data of Australian inland water bodies to monitor water toxicity level caused by algal blooms."

It aims to acquire data across the VNIR and SWIR wavelengths and in particular the specific wavelengths for early detection of algal blooms over the MDB. HISA aims to have a mission operation lifetime of 6 years where it prioritises imagery of Australian land and water bodies. After this period, it will then expand its imaging capabilities to the rest of the world and allow for international collaboration focusing on other global environmental issues. This mission is also accompanied by a secondary EO mission which aims to investigate anomalies and discrepancies in the hyperspectral data by providing high resolution color images.

The primary mission also consists of an engineering mission component which is defined as:

"The demonstration of a spacecraft bus which is capable of high data throughput for maximum utility and high level of onboard autonomy."

To fulfil the high data throughput and downlink capability, HISA will utilise hardware such as a deployable X-band phased array antenna using 16-QAM scheme and software processes such as lossless compression. Approximately a total of 70-80 hyperspectral images will be taken over Australia on a daily basis which is then stored on the Solid State Mass Memory (SSMM) for downlink to the ground stations.

HISA aims to demonstrate a high level on on-board autonomy to support the components and to realize the high data throughput capabilities. The Command and Data Handling $(\mathrm{CDH})$ subsystem is mainly responsible for the execution of autonomous processes and is discussed in more depth in Section 5.

\subsection{Secondary mission}

The optical RGB camera serves the purpose of a secondary EO mission with two different shooting modes: Single Point Tracking (SPT) mode and Multi-Point Continuous (MPC) mode. This mission mode will not be used frequently during the mission life of HISA as the payload is a redundant payload. These modes aim to provide additional data and validate imagery captured during the primary mission only when necessary. It will be used in the presence of any anomaly in the hyperspectral data from or in the failure of the primary payload.

The SPT mode consists of HISA pointing at a stationary target on the ground and executes attitude stabilisation maneuvers to capture photographs of the same target from three different angles: $-20^{\circ}$ to the target, once at nadir, $+20^{\circ}$ to the target. Capturing images of the same target in quick succession allows for comparison of a particular site and has potential disaster monitoring applications. In the MPT mode, HISA will have multiple targets on the ground and take continuous photographs of these various targets.

\subsection{Success criteria}

The success of this mission is dependent on a few factors: the successful transmission of scientific data from both all payloads, demonstration of high level autonomy, length of service beyond lifetime of mission. It is based on a point system where points of different values are awarded for successful execution or operation of tasks. Table 1 shows a list of criteria and respective points which represent the different accomplishments of HISA during the mission lifetime. Minimum success is defined as establishing a successful communication link between the spacecraft and ground station with telemetry downlink. Partial success is defined as successful execution of the primary mission and data downlink of at least one hyperspectral image. For full success, emphasis is placed on demonstration of autonomous operation and maximum data throughput capabilities of the spacecraft. After the original mission lifetime of 5 years, HISA will go into extended mission mode and continue to perform observations and data transmission.

Table 1. The list of criteria representing different accomplishments during the mission lifetime.

\begin{tabular}{lc}
\hline Success Criteria & Points \\
\hline Communication link and telemetry downlink & 50 \\
Secondary EO mission & 100 \\
Primary mission execution \& data downlink & 150 \\
Automatic target acquisition and observation & 200 \\
Downlink of 25 images or more per orbit & 200 \\
Meaningful hyperspectral data analysis & 300 \\
\hline
\end{tabular}

\subsection{Orbit analysis}

According to the guidelines set out by the Inter-Agency Space Debris Coordination Committee (IADC), spacecraft in LEO must be able to re-enter the atmosphere and deorbit within 25 years after the end of mission life for orbital debris mitigation. ${ }^{13)}$ Additionally, there are no orbital control or on-board propulsion systems for station keeping on HISA meaning that the spacecraft will decay naturally until it re-enters and burns up in the atmosphere. To meet the guidelines by IADC, a circular Sun-Synchronous Orbit (SSO) between 600-700 km is investigated.

SSOs are favourable for EO and remote sensing spacecraft as it provides consistent lighting conditions for power generation and imagery applications. A few conditions required for the primary mission includes: consistent illumination for comparative imaging, early passover time over target, appropriate altitude to achieve sufficient ground sampling resolution and relatively short revisit time. Some of these parameters are driven by the hyperspectral camera specifications and a trade-off was required to choose the best orbit for maximum coverage. For these reasons, the desired orbit is a $650 \mathrm{~km}$ circular SSO with an inclination of 97.8 degrees and local descending time of approximately 10:00 am. This particular orbit has a revisit time of approximately 6 days but this can be reduced by utilising the tilting capability of the satellite during the primary mission. 
The orbital path was simulated using System Tool Kit (STK) over Australia and is shown in Fig. 3

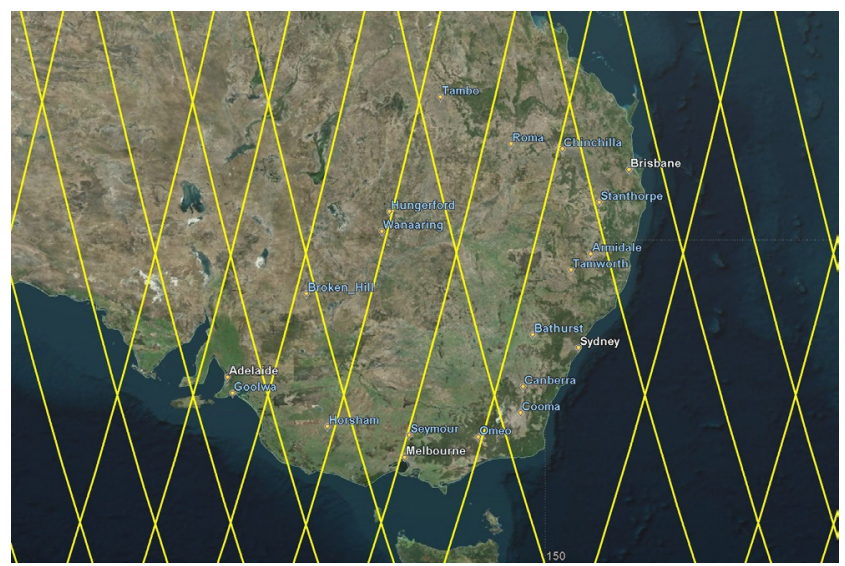

Fig. 3. Ground coverage of HISA over the MDB in a 6 day period simulated on System Tool Kit (STK).

\subsection{Launch opportunities}

An advantage of microsatellite development is the readily accessible number of launches and launch vehicles where the microsatellite can 'piggyback' a launch into orbit. Users who decide to 'piggyback' on a launch vehicle with a main payload may not necessarily be able to choose a specific orbit but the increasing number of launches per year allow piggyback launch users to choose an orbit range close to their target.

More recently, in response to the growing capability of launch small satellites, the European Space Agency (ESA) has announced an opportunity for a piggyback launch for microsatellites onboard the Vega launcher as part of the Small Spacecraft Mission Services program. ${ }^{14)}$ The Vega is able to launch multiple small satellite payloads and can carry up to $1500 \mathrm{~kg}$ to a $500-800 \mathrm{~km}$ sun synchronous orbit. Figure 4 shows the Vega launcher carrying four small satellites with dimensions $80 \times 80 \times 100 \mathrm{~cm}$ weighing between $80-150 \mathrm{~kg}$. The proposed specifications of HISA conform to the Class 2 spacecraft constraints defined by ESA and thus this launch opportunity is very desirable. HISA will target launch of the flight model by 2023 .

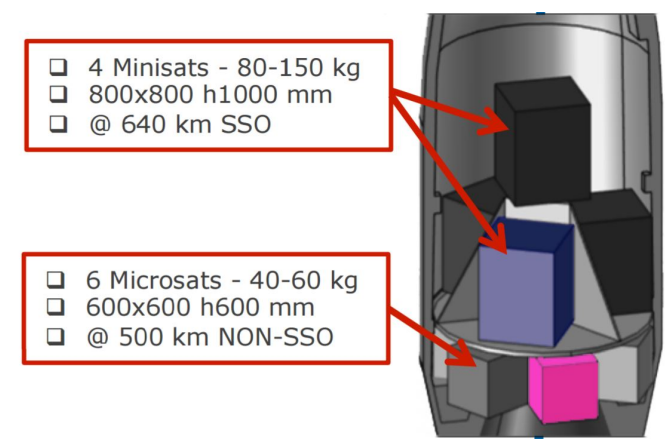

Fig. 4. One particular configuration using the ESA Vega Launcher to deploy small satellites into SSO.

\section{The Hyperspectral Camera}

The hyperspectral camera is the primary payload is the most important component of the whole spacecraft and thus the spacecraft bus, structure, and system is designed around to support this payload. Due to the commercial nature of the mission in this study it is essential to avoid a single point failure caused by the hyperspectral camera. Also, for risk mitigation purposes, the design and development of the hyperspectral camera is assigned to a US company, Brandywine Photonics, who specialise in manufacturing custom hyperspectral cameras for specific customer needs. Table. 2 shows a list of specifications for the hyperspectral camera based on an orbit of $650 \mathrm{~km}$.

Table 2. Summary of the hyperspectral camera design technical specifications.

\begin{tabular}{|c|c|}
\hline Specification & Value \\
\hline Sensor type & Dyson pushbroom spectrometer \\
\hline & CMOS ROIC \\
\hline \multirow{2}{*}{ Spectral Range } & $\operatorname{VNIR}(380-980 \mathrm{~nm})$ \\
\hline & SWIR(980-2500 nm) \\
\hline FPA & $4096 \times 512 \mathrm{px}$ \\
\hline VNIR spectral resolution & $10 \mathrm{~nm}$ \\
\hline SWIR spectral resolution & $5 \mathrm{~nm}$ \\
\hline Spectral channels & 260 bands \\
\hline GSD & $10 \mathrm{~m} / \mathrm{px}$ \\
\hline Swath & $40 \mathrm{~km}$ \\
\hline \multirow[t]{3}{*}{ SNR } & @ 380 nm: 150 \\
\hline & @ 500 nm: 300 \\
\hline & @ 1680 nm: 100 \\
\hline Telescope optics & $\begin{array}{l}\text { Ritchey Chretien w/ 2-mirror } \\
\text { freeform corrector }\end{array}$ \\
\hline Telescope material & SiC or Be composite \\
\hline Length & $\approx 600 \mathrm{~mm}$ \\
\hline Aperture & $600 \mathrm{~mm}$ \\
\hline F-stop & 1.8 \\
\hline Focal length & $900 \mathrm{~mm}$ \\
\hline IFOV & $16.67 \mu \mathrm{rad}$ \\
\hline Dark current density & $0.5 \mathrm{~A} / \mathrm{m}^{2}$ \\
\hline Power consumption & $30 \mathrm{~W}$ \\
\hline Total estimated mass & $30 \mathrm{~kg}$ \\
\hline Frame rate & $125 \mathrm{~Hz}$ \\
\hline Data rate & $931.84 \mathrm{Mbps}$ \\
\hline Quantisation & 14 bits/px \\
\hline
\end{tabular}

A Dyson pushbroom spectrometer (Fig. 5) is used where a single line of the target area perpendicular to the orbital flight path is fed through a slit into the diffraction grating. The diffraction grating efficiency for this particular design has a peak of $88 \%$. This one dimensional light is then converted into a two dimensional image line by line using a Complementary MetalOxide Semiconductor (CMOS) Read Out Integrated Circuit (ROIC) as the spacecraft flies over the target.

The spatial specifications are designed around a $650 \mathrm{~km}$ orbit and is important as the resolution must be fine enough to identify and evaluate the rivers in the MDB whilst capturing a wide area for any meaningful analysis. The rivers which form the MDB extend across three states and measure $3375 \mathrm{~km}$. The channel width of these rivers vary widely and is sampled to determine a suitable Ground Sampling Distance (GSD) and swath for the camera. A GSD of $10 \mathrm{~m}$ is required ensure that the camera can resolve the rivers and water bodies of interest in the MDB as well as achieving sufficient pixel or sub-pixel spatial resolution of smaller creeks and dams for animal drinking. A 


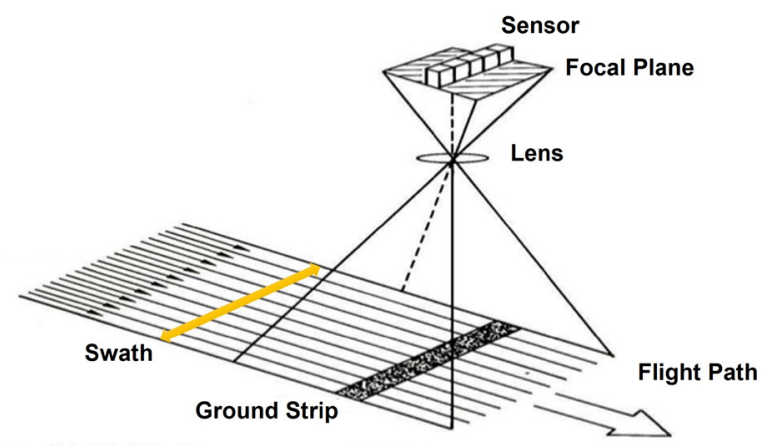

Fig. 5. Pushbroom scanning works by repeatedly capturing thin ground strips as the spacecraft flies over the target on the ground.

swath width of $40 \mathrm{~km}$ is desired to capture a wide portion of the MDB without having to make multiple passes which may take several days depending on the orbit.

To accommodate the wide spectral range, two separate sensors will be utilised: a VNIR sensor from 380-980 $\mathrm{nm}$ and a SWIR sensor from $980-2500 \mathrm{~nm}$. The VNIR sensor is a Silicon based detector while the SWIR sensor is a Mercury Cadmium Telluride (MCT) type II superlattice based detector. The spectral resolution for the VNIR sensor is $10 \mathrm{~nm}$ which is sufficient to detect blue-green algal growth in the MDB. The SWIR sensor aims to have a $5 \mathrm{~nm}$ resolution to acquire fine information in surveillance and mineral mapping applications.

A high SNR is particularly important for remote sensing missions as hyperspectral sensors onboard spacecraft at an orbit of $700 \mathrm{~km}$ receive approximately $0.01 \%$ (10000 times less) of the radiation received by sensors mounted on airborne systems at an altitude of $5 \mathrm{~km} .{ }^{11)}$ Maximising the SNR especially in the SWIR region takes highest priority in most remote sensing missions. This is heavily dependent on the physics and optical design of the payload. The SNR for the system is shown in Table. 2 but over time, the aim is to increase the SNR to approximately 500-600:1.

A wide aperture is used to increase SNR especially when passing over low albedo ocean paths and to further increase SNR in the SWIR detection, HISA will execute a 'nodding' maneuver to increase the exposure time when passing over a certain target. This camera has a frame rate of $125 \mathrm{~Hz}$ and thus will cover $55 \mathrm{~m} / \mathrm{px}$ (assuming orbital velocity of $25000 \mathrm{~km} / \mathrm{hr}$ ). To achieve a $10 \mathrm{~m} \mathrm{GSD}$, the spacecraft will need to rotate around the axis perpendicular to the longitudinal track of the orbital flight path to expose a $10 \mathrm{~m}$ ground track distance every 8 milliseconds (for $125 \mathrm{~Hz}$ ) which equates to a 5:1 'nodding' angular rotation rate. A higher nodding ratio subsequently increases the SNR.

Wavelengths beyond the visible spectrum $(780 \mathrm{~nm}$ and above) are much more susceptible to noise and require cooling to achieve the desired level of SNR. The sensors are have passive cooling where it is thermoelectrically cooled using the Peltier effect ${ }^{12}$ ) to maintain the temperature below a certain level. However, the SWIR CMOS sensor may require mechanical cyrocooling to keep the temperature of the sensor below 150 $\mathrm{K}$ for dark current suppression especially in the higher wavelengths.

In order to adhere to the microsatellite class category, the only physical constraint for the hyperspectral camera is for the whole payload to fit within the volume of the microsatellite structure/frame of 80 (b) $\times 80$ (b) $\times 100(\mathrm{~h}) \mathrm{cm}$. The hyperspectral camera and sensor itself is mounted on a central cylinder modified Ritchey-Chretien Cassegrain Telescope with Chrisp 2-mirror freeform corrector optical bench with and aperture diameter of $600 \mathrm{~mm}$ and length of $600 \mathrm{~mm}$.

\subsection{High resolution optical camera}

In the event of any failure in the primary payload or in executing the primary mission, HISA houses a high resolution optical camera in the RGB spectrum as a secondary payload which also acts as a redundant payload. Access to high resolution satellite imagery coupled with hyperspectral imaging capabilities would increase accuracy and be able to provide rapid updates for land mapping and disaster monitoring applications. At this stage, the supplier and manufacturer of the camera has not been determined but a camera with a GSD of $<5 \mathrm{~m}$ at nadir for $20-40 \mathrm{~km}$ swath at $650 \mathrm{~km}$ is desired.

\section{Satellite Structure and Subsystems}

As the launch vehicle has not yet been determined, the specific acceleration, vibrational and acoustic loads the spacecraft must endure during launch are currently unknown which makes it challenging to design the specific shape and structure of the spacecraft. However, the overall size of the spacecraft bus and structure is proposed to be $80 \times 80 \times 100 \mathrm{~cm}$ with a launch mass of approximately $100 \mathrm{~kg}$.

All deployable appendages are attached to the spacecraft via a spring loaded hinge and are stowed away with a Kevlar cord during pre-flight testing and in the pre-launch configuration. The deployment mechanism consists of a pyrotechnic cutter or a thermal knife which cut the Kevlar cords in tension holding the deployable appendages while in the stowed configuration. The cutting command is automatically executed by the OBC during spin-stabilisation of the spacecraft and after separation from the launch vehicle. The deployment of the appendages are critical to the success of the mission and for the life of the spacecraft as it primarily relies on solar energy for power generation. Thus the system is fully redundant with multiple independent cutters, knives, circuits and commands.

\subsection{Attitude determination and control subsystem}

The Attitude Determination and Control Subsystem (ADCS) consists of a combination of actuators and sensors to maneuver the spacecraft and maintain a certain orientation during science missions. The attitude control system is made up of a triaxial magnetorquer for coarse attitude control and four reaction wheels for fine attitude control. The sensors include six sun sensors and three mangnetometers (one for each axis) for coarse attitude determination and two star trackers and a MEMS gyroscope for fine attitude determination. A GPSR is used for orbit determination but there are no thrusters or on-board propulsion for orbit control.

The magnetorquers are primarily responsible for detumbling and stabilising the spacecraft after separation from the launch vehicle and also helps maintain the spacecraft attitude during safe mode and normal/standby mode. The reaction wheels are the primary attitude actuators used in the primary mission to provide more rapid and accurate attitude pointing for EO. It 
provides 3-axis attitude control for high accuracy nadir pointing and fine slewing during shutter exposure.

In context of the attitude pointing requirements and considering the constraints of the hyperspectral camera, an absolute pointing accuracy of 100 arcseconds is required with a 'nodding' rate of 5:1. Additionally, to increase the SNR and avoid image blurring, a short term relative pointing stability of 5 arcseconds over 10 seconds of sensor exposure is required during any imaging mode a frame rate of $125 \mathrm{~Hz}$ for the hyperspectral camera. This is difficult for a satellite with a bus size such as HISA due to the small inertia but not impossible to acheive. PROBA $^{17)}$ has previously demonstrated pointing accuracy of 1 arcminute on a satellite bus smaller than HISA.

\subsection{Communication subsystem}

The S-band (2-4 GHz) will be utilized for housekeeping and TT\&C data transmission and reception. This data includes the spacecraft status and health, and gives information about whether the spacecraft is operating nominally. It is also used to diagnose any faults on the spacecraft. TT\&C uplink from the ground station involves commands which control the spacecraft functioning. As this can be used to reset spacecraft systems, reconfigure various settings and request science mission data download, the uplink connection needs to be protected using secure encryption to prevent access by any unauthorised or third-parties.

To downlink the science mission data, the X-band (8-12 $\mathrm{GHz}$ ) frequency is used for its reliable and high bandwidth capabilities. To realize this, an $80 \times 80 \mathrm{~cm}$ deployable X-band phased array antenna system is utilised. The deployable panel is a flat grid consisting of a $12 \times 12$ square array of 144 radiating elements where the phase of each signal and element can be shifted and combined to produce a higher gain (Fig. 6). The $\mathrm{X}$-band phased array antenna system has already been tested and validated previously on the NASA EO-1 satellite ${ }^{16)}$ and ESA GAIA spacecraft. ${ }^{18)}$ A few advantages of using the $\mathrm{X}$-band is listed below:

- No signal attenuation from 'rain fade'.

- Lower pointing accuracy requirements compared to highgain antenna dishes.

- Resistant to additional torque disturbances.

- Robust to single point failure.

- No additional mechanical or moving components.

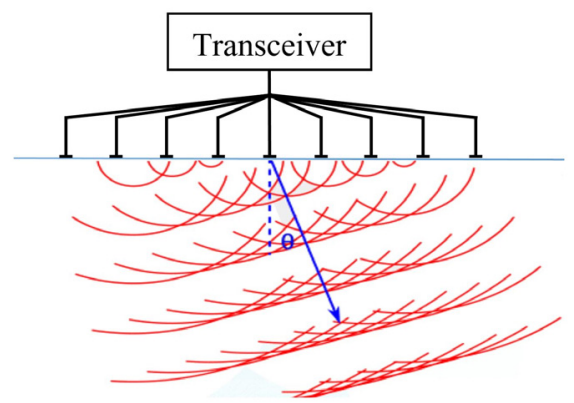

Fig. 6. A phased array antenna steers the beam electronically by shifting the phase of each signal which increases the gain and transmission capability.

The science data stored on the 1 TB SSMM is compressed and encoded before being sent to the $\mathrm{X}$-band upconverter and then to the X-band phased array antenna. By utilising 16-QAM (Quadrature Amplitude Modulation) with down conversion at the baseband, HISA aims to transmit at $800+$ Mbps for a total of 336 Gigabits per pass for each ground station assuming 7 minutes of effective communication link. By utilising three ground stations in Australia located at Sydney, Darwin and Perth, total transmission volume can be increased to over 1 Terabit or 125 GB per day.

\subsection{Electrical power subsystem}

Power is generated by Gallium Arsenide (GaAs) solar cells mounted to one side of the spacecraft and also on two additional deployable panels such that it triples the sunlight illumination surface area. Each surface/panel measure $80 \times 100 \mathrm{~cm}$ and are completely covered in solar cells to produce approximately $400-450 \mathrm{~W}$ of power at an efficiency of $28.8 \% .^{19)}$ It aims to supply sufficient power to execute all the mission modes while charging the battery so that the spacecraft does not shut down during the eclipse phase of the orbit when sunlight is not available. Achieving the highest efficiency of the cells is also another important goal and a widely adopted method to maximise the efficiency is the use of Maximum Power Point Tracking (MPPT) algorithms where the Electrical Power System ensures that the solar cells are consistently operating at the peak of the PV curve.

To prevent spacecraft shutdown during the eclipse phase of the orbit, lithium ion batteries provide passive common DC bus voltage to the spacecraft. The batteries are charged by the GaAs solar cells via a DC-DC converter which operates in the MPPT mode or the constant voltage mode in cases where the battery charge is full and load demand is low. Another phase of the spacecraft mission which heavily relies on the battery as a source of power is after separation from the launch vehicle. It is crucial that HISA is able to deploy its SAPs and point to the Sun before depletion of the batteries. Once the launch vehicle and orbit injection parameters are determined, a detailed power budget will be conducted. Simulations will also be conducted to calculated the maximum allowable time HISA has to point to the Sun before reaching a critical depth of discharge of the batteries.

\subsection{Command and data handling subsystem}

The Command and Data Handling ( $\mathrm{CDH})$ subsystem is overseen by the spacecraft $\mathrm{OBC}$ and is responsible for the overall functioning of the spacecraft (Fig. 7). It distributes commands received from the ground station to the respective subsystems and ensures nominal operation of the spacecraft at all times. Figure 7 shows the connection to each component and the interaction of the $\mathrm{CDH}$ and the various subsystems. The $\mathrm{CDH}$ consists of the: flight computer hardware which employs a Field Programmable Gate Array (FPGA) as it is flexible and reliable; the flight software; and a 1 TB Solid State Mass Memory (SSMM) drive for data storage.

Part of the primary engineering mission is the demonstration of high level of onboard autonomous processes to reduce human input. The decrease in interaction from the ground segment also reduces the chance of error due to human factors. It aims to demonstrate the following automatic functions on-orbit:

- FDIR implementation - to ensure the continuation of safe operation in the event of a fault. By the time human inter- 


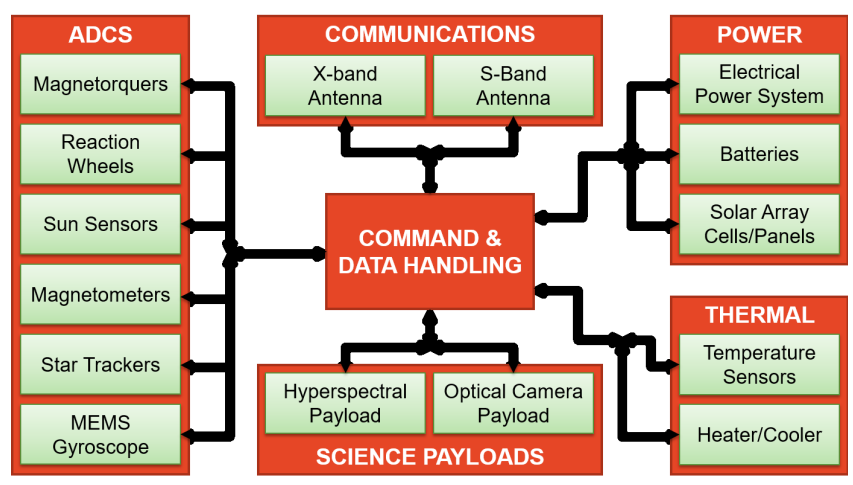

Fig. 7. The basic interface diagram showing the interaction of the $\mathrm{CDH}$ and various components of the subsystems.

vention is required, the system may have already sustained irreversible damage. HISA will reset and reconfigure the spacecraft accordingly.

- Mission scheduling, preparation and execution - predetermined target coordinates are uplinked to HISA and the OBC will calculate if targets are within FOV threshold to begin imaging. HISA will also set up all instruments required and perform necessary attitude maneuvers.

- Data storage and management - All the data acquired from the primary and secondary EO missions will be stored and managed autonomously, and lossless compression will be applied when necessary.

- Data communication link - Will automatically establish a communication link to dump the data stored on the SSMM if the orbital path coincides with a ground station. This is useful when human presence is unavailable.

- Resource and performance management - Allocates and distributes electrical power to subsystems according to load demand and then evaluate the performance of HISA and all its subsystems.

The additional information from an extra dimension in the hyperspectral datacube comes at a steep price of increased datasize and is often known as 'the curse of dimensionality' which presents a challenge to microsatellites with limited bandwidth capabilities. It creates a bottleneck for efficient transmission of the mission data to the ground. Considering the hyperspectral camera in this study, a single image consisting of 260 spectral bands, 4096 by 2048 pixels, and 14 bits per image results in an image size of 30.5 Gigabits. This allows for only 10 images to be downlinked per pass given a strong and stable communication link. For comparison, HyspIRI produces 1 Terabit of data per orbit ${ }^{20)}$ and HISUI (onboard ALOS-3) produces 11.52 Terabits daily. ${ }^{21)}$

To counter this issue, lossless compression is able to reduce the data size while retaining image quality and thus an FPGA implementation of the Fast Lossless compression algorithm ${ }^{22}$ ) is utilised onboard HISA. It has been shown to produce the best compression ratio (3.1:1) and low bits/sample when compared to other compression algorithms. ${ }^{23), 24)}$

\section{Conclusion}

The University of Sydney is proposing the design of a commercially viable $100 \mathrm{~kg}$ class microsatellite HISA - Hyper- spectral Imaging Satellite for Australia. The spacecraft is $80 \times 80 \times 100 \mathrm{~cm}$ in size and aims to address the compelling agricultural and environmental problems specific to the Australian economy by analysing farmland and the water quality of inland lakes and rivers. It also aims to increase space activity and development within Australia.

The design improves upon previous hyperspectral missions by optimising the system for maximum data utility and throughput. The spacecraft structure is built to support the hyperspectral camera payload and has two deployable solar array panels and an extra deployable panel dedicated to a X-band phased array antenna. HISA will also attempt to utilise onboard autonomy of processes during science missions and in automatic fault detection, isolation and recovery. HISA hopes to respond to the significant need for a hyperspectral remote sensing platform dedicated for specific Australian needs.

\section{Acknowledgments}

The Author would like the acknowledge members of the HISA development team for their contributions to the project. A special mention to Jalal Elwazze for developing the CAD model. The author would also like to thank Nina and Sara for their support and guidance.

\section{References}

1) Vane, G., Goetz, A. F., and Wellman, J. B.: Airborne Imaging Spectrometer: A New Tool for Remote Sensing, IEEE Transactions on Geoscience and Remote Sensing, 6 (1984), pp. 546-549.

2) Sims, N. C., Culvenor, D., Newnham, G., Coops, N. C., and Hopmans, P.: Towards the Operational Use of Satellite Hyperspectral Image Data for Mapping Nutrient Status and Fertilizer Requirements in Australian Plantation forests. IEEE Journal of Selected Topics in Applied Earth Observations and Remote Sensing, 6 (2013), pp. 320-328.

3) Buchen, E. and DePasquale, D.: 2016 Nano/Microsatellite Market Assessment, SpaceWorks Enterprises, Inc. (SEI), 2017.

4) Organisation for Economic Cooperation and Development Space Forum, http://www.oecd.org/futures/oecdspaceforum.htm (accessed March 24th, 2017).

5) Space Policy Unit: Analysis of Australian Government Space Activities, Dept. Innovation, Industry, Science and Research, Australia, 2010 .

6) Held, A., Clayfield, K., Ward, S., Dyke, G., and Harrison, B.: Continuity of Earth Observation Data for Australia: Research and Development Dependencies to 2020, 2012, https://doi.org/10.4225/08/584d95f793de2 (accessed March 25th, 2017).

7) Bowling, L. C. and Baker, P. D.: Major Cyanobacterial Bloom in the Barwon-Darling River, Australia, in 1991, and Underlying Limnological Conditions, Marine and Freshwater Research, 47 (1996), pp. 643-57.

8) NSW Office of Water: Assessment of the Socio-economic Impacts of Murray River Blue-green Algae, Earnst \& Young Australia, 2010.

9) Lin, S., Borstad, G. A., and Gower, J. F. R.: Remote Sensing of Chlorophyll in the Red Spectral Region, Elsevier Oceanography Series, 38 (1984), pp. 317-336.

10) MacColl, R. and Guard-Friar, D.: Phycobiliproteins, CRC, 1986.

11) Kumar, L., Schmidt, K., Dury, S., and Skidmore, A.: Imaging Spectrometry and Vegetation Science, Imaging Spectrometry, 4 (2002), pp. 111-155.

12) Bell, L. E.: Cooling, Heating, Generating Power, and Recovering Waste Heat with Thermoelectric Systems, Science, 321 (2008), pp. 1457-1461.

13) IADC Space Debris Mitigation Guidelines, IADC-02-01 Revision 1, 
Inter-agency Space Debris Coordination Committee, 2007.

14) Bianchi, S. and VEGA Integrated Project Team: VEGA, the European Small Launcher: Development Status, Future Perspectives, and Applications, Acta Astronautica, 63 (2008) pp. 416-427.

15) Stewart, A. C.: A New and Innovative Use of the Thermal Knife and Kevlar Cord Components in a Restraint and Release System, 9th European Space Mechanisms and Tribology Symposium, 2001, pp. 231238.

16) Perko, K., Huggins, R., Heisen, P., Miller, G., McMeen, D., Dod, T., Staren, J., Sank, V., and Nguyen, X.: X-Band Phased Array Antenna Validation Report, NASA, 2002, pp. 2-4.

17) Teston, F., Vuilleumier, P., Hardy, D., Tilmans, E., and Gantois, K.: Proba Proves the Technology-Proba-1 Passes 5 years in Orbit, ESA bulletin, 129 (2007), pp. 48-53.

18) Allica, J. C., Alonso, E., Amado, M., Bazán, A., Casares, F., de Witte, E., Garcia, Q., McConnell, T., Montesano, A., Santilario, J., and Serrano, J. L.: Architecture of Gaia Satellite Phased Array Antenna, IEEE Proceedings of the Fourth European Conference on Antennas and Propagation, 2010, pp. 1-4.

19) Green, M. A., Emery, K., Hishikawa, Y., Warta, W., and Dunlop. E. D.: Solar Cell Efficiency Tables (Version 49), Progress in Photovoltaics: Research and Applications, 25 (2017), pp. 3-13.

20) Oaida, B.: HyspIRI Mission Concept, 2012, NASA Workshop, p. 2.

21) Hihara, H., Moritani, K., Inoue, M., Hoshi, Y., Iwasaki, A., Takada, J., Inada, H., Suzuki, M., Seki, T., Ichikawa, S., and Tanii, J.: Onboard Image Processing System for Hyperspectral Sensor, Sensors, 15 (2015), pp. 24926-24944.

22) Klimesh, M.: Fast Lossless Compression of Multispectral-Image Data, NASA Tech Brief, 2006, pp. 6-7.

23) Pizzolante, R. and Carpentieri, B.: Multiband and Lossless Compression of Hyperspectral Images, Algorithms, 9 (2016), p. 16.

24) Rizzo, F., Carpentieri, B., Motta, G., and Storer, J. A.: Lowcomplexity Lossless Compression of Hyperspectral Imagery via Linear Prediction. IEEE Signal Processing Letters, 12 (2015), pp. 138141. 